\title{
Metal Enrichment Processes in the ICM - Starbursts and Galactic Winds
}

\author{
W. Kapferer ${ }^{1}$, T. Kronberger ${ }^{1}$, W. Domainko ${ }^{1}$, S. Schindler ${ }^{1}$, \\ E. van Kampen ${ }^{1}$, S. Kimeswenger ${ }^{1}$, M. Ruffert ${ }^{3}$, M. Mair ${ }^{1}$, \\ and D. Breitschwerdt ${ }^{2}$ \\ ${ }^{1}$ Institut für Astro - und Teilchenphysik, Innsbruck, Austria \\ ${ }^{2}$ Institut für Astronomie, Universität Wien, Austria \\ ${ }^{3}$ Department for Mathematics and Statistics, University of Edinburgh, UK
}

\begin{abstract}
We present an investigation of the metal enrichment of the intra-cluster medium (ICM) by galactic winds and merger-driven starbursts. We use combined N-body/hydrodynamic simulations with a semi-numerical galaxy formation model. The inhomogeneities in the metal distribution caused by these processes are an ideal tool to reveal the dynamical state of a galaxy cluster. We show that X-ray weighted metal maps distinguish between pre- or post-merger galaxy clusters by comparing the metallicity distribution with the galaxy-density distribution: pre-mergers have a metallicity gap between the subclusters, post-mergers a high metallicity between subclusters.
\end{abstract}

\section{Reference}

Kapferer, W., et al. 2006, A\&A, 447, 827.

\section{Acknowledgements}

We acknowledge the support by the bm:bwk Austrian Grid (Grid Computing) Initiative and the Tiroler Wissenschaftsfonds (Gefördert aus Mitteln des vom Land Tirol eingerichteten Wissenschaftsfonds).

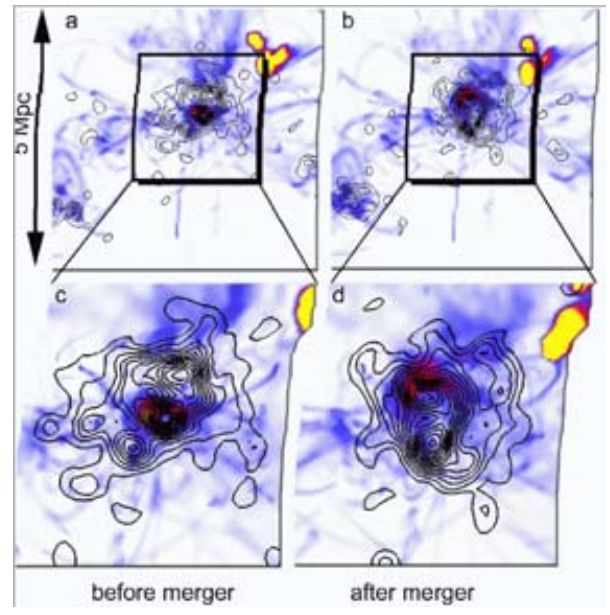

Artificial X-ray weighted metallicity maps of a model cluster with galaxy-density isolines drawn in. The upper images show a $(5 \mathrm{Mpc})^{2}$ region, whereas the lower images are the magnifications of the innermost grid $(2.5 \mathrm{Mpc})^{2}$. The metallicity gap before the first encounter of the subcluster with the main cluster is clearly visible. The main cluster is located in the center of the image (c) and the gap lies above. 